\title{
Valoración ética de la Modernidad según Alasdair Maclntyre*
}

\section{(Ethical assessment of Modernity according to Alasdair Maclntyre)}

\author{
JORGE MARTÍN MONTOYA CAMACHO \\ Universidad de Navarra, Pamplona \\ jmontoya.1@unav.es \\ ORCID 0000-0002-6924-7154
}

Resumen: ¿Puede ser comprendida la moralidad aisladamente de los órdenes sociales, culturales, políticos y económicos en los que surge y se desarrolla? ¿El interés intelectual por la moralidad de nuestro tiempo requiere algo más que el análisis de teorías éticas? Las respuestas de Alasdair Maclntyre a estas preguntas se muestran a través del trabajo de toda una vida, en la que ha desarrollado un análisis ético de la Modernidad. El libro «Valoración ética de la modernidad según Alasdair Maclntyre», de Hernando José Bello Rodríguez y José Manuel Giménez Amaya, expone el desarrollo de tal análisis, llevado a cabo por el filósofo escocés en el marco de sus ideas que van desde la publicación de su obra After Virtue, hasta la reciente aparición de Ethics in Conflicts of Modernity.

Palabras clave: teleología, racionalidad, emotivismo, individualismo, sociedad.

Abstract: Can morality be understood in isolation from the social, cultural, political and economic orders in which it arises and develops? Does the intellectual inte-

Bello Rodríguez, H. J. \& Giménez Amaya, J. M. 2018. Valoración ética de la modernidad según Alasdair MacIntyre. Pamplona: EUNSA. 
rest in morality of our time require more than the analysis of ethical theories? Alasdair Maclntyre's answers to these questions are shown through a lifetime's work, in which he has developed an ethical analysis of Modernity. The book "Ethical assessment of modernity according to Alasdair Maclntyre", by Hernando José Bello Rodríguez and José Manuel Giménez Amaya, exposes the development of such an analysis, carried out by the Scottish philosopher within the framework of his ideas ranging from the publication of his After Virtue, until the recent appearance of Ethics in Conflicts of Modernity.

Keywords: teleology, rationality, emotivism, individualism, society.

El filósofo alemán Robert Spaemann indicó en su libro Ética. Cuestiones fundamentales (2010), que la distinción entre las teorías éticas naturales y las no-naturales se encuentra en que, mientras la primeras se dirigen a la indagación del bien supremo al que pueden aspirar las tendencias humanas, las segundas suelen enfocarse, simplemente, en tratar de averiguar lo que es moralmente justificado en una sociedad. Para este filósofo, las éticas naturales ayudarían a alcanzar lo que el ser humano quiere de verdad, y en el fondo de sí mismo, mientras que las de orden no-natural, al no conseguir este cometido, se verían abocadas, de una manera $\mathrm{u}$ otra, a utilizar elementos represivos para conseguir una determinada disposición social en los individuos. A la base de esta cuestión, se encontraría la incapacidad de algunos sistemas normativos de ofrecer al sujeto modos de superar libremente sus propios caprichos, y de no verse sometidos a las manipulaciones y deseos de otros (Spaemann 2010, 37).

La certera clasificación de Spaemann se ve llena de contexto y de un adecuado significado a través de la idea de «emotivismo» del filósofo escocés Alasdair MacIntyre, capital en su crítica a la Modernidad. Para MacIntyre, el emotivismo deja de lado cualquier distinción auténtica entre las relaciones sociales manipuladoras y las no manipuladoras. Esto es debido a que, en éste, los juicios de valor son tomados por el sujeto como expresiones de sus propios sentimientos y actitudes, y que son -a la veztendentes a transformar los sentimientos y actitudes de otros (MacIntyre 1987, 40-41). 
Dentro de la dinámica emotivista, para MacIntyre, el ser humano se ve incapacitado para apelar en verdad a criterios impersonales, aunque el sujeto, junto con otros, pueda llegar a pensar que lo hace. Por tanto, la única realidad que distingue este tipo de discurso moral es la tentativa de una voluntad, individual o colectiva, de poner de su propio lado las leyes, actitudes, sentimientos, preferencias y elecciones que implican a los otros sujetos de la sociedad. De este modo los demás individuos serán siempre medios, y nunca fines, de las decisiones morales del ser humano (MacIntyre 1987, 40-41).

El libro «Valoración ética de la Modernidad según Alasdair MacIntyre» de Hernando José Bello Rodríguez y José Manuel Giménez Amaya (EUNSA 2018), nos presenta esta crítica del filósofo anglosajón al mundo moderno desde la perspectiva de la Ética. Desde las primeras páginas observamos que tal crítica está muy relacionada con la trayectoria vital e intelectual de MacIntyre. Los autores del libro lo dejan muy claro, y con esto aportan la trama adecuada que permite comprender el pensamiento del emérito profesor de la University of Notre Dame. Por este motivo, al reseñar, comentar y valorar esta obra, utilizaré el primer capítulo como hilo conductor para las ideas que aparecen en los otras dos partes de este libro.

La exposición del itinerario vital muestra cómo MacIntyre ha afrontado, de modos personal y filosófico, los debates contemporáneos. Resaltan algunos puntos significativos de su vida intelectual y académica, entre los que se encuentran sus primeros encuentros con la filosofía aristotélico-tomista siendo estudiante del Queen Mary College de la Universidad de Londres (Bello Rodríguez \& Giménez Amaya 2018, 25-28)․․ MacIntyre no se adhirió inmediatamente a tal línea de pensamiento, ya que en su juventud no encontró argumentos concluyentes que lo llevaran a abrazarla. Años más tarde se percatará de que el problema de su indecisión no se encontraba en el tomismo, sino en el modo propio de funcionar de los debates de la filosofía académica del siglo XX, en la que siempre es posible encontrar objeciones a cualquier conjunto de tesis defendidas, y que él ha-

1 En adelante citaré sólo las páginas de Bello Rodríguez, H. J. \& Giménez Amaya, J. M. 2018. Valoración ética de la modernidad según Alasdair MacIntyre. Pamplona: EUNSA. 
bía incorporado, de un modo excesivamente teórico, en su aproximación intelectual a las diferentes cuestiones de la vida humana.

Desde las primeras páginas del libro nos encontramos con la presentación del «expresivismo» -también llamado «expresivismo emotivista», o simplemente «emotivismo", como ya se ha mencionado- como una de las posturas más combatidas por MacIntyre (pp. 29-30 y 42-50). El filósofo británico llegó a conocer esta forma de pensar la Ética a partir de sus análisis de las semejanzas de dos posturas filosóficas que, a primera vista, parecen no presentar similitudes. Estas son el existencialismo francés de Jean-Paul Sartre y el positivismo lógico de Alfred J. Ayer. Sin embargo, para MacIntyre, es posible observar cómo las visiones sobre las decisiones morales de ambos filósofos se acercan entre sí, si se tiene en cuenta el principio que las informa.

El estudio de MacIntyre sobre el «emotivismo» parece indicar que tal postura moral se fundamenta en tres etapas que evidencian un marco teórico subjetivista que puede dejar al «yo» a merced de relaciones sociales manipuladoras. En la primera etapa se establece un factor subjetivo decisorio y valorativo, por el que se indica que el razonamiento práctico de cualquier individuo obtiene sus conclusiones de las premisas que el propio sujeto ha elegido previamente, y que éste estima que aquello que hace que una razón sea buena o mala o, cuando menos, suficiente para llevar a cabo una acción, es la propia decisión del sujeto (p. 29). La segunda etapa introduce el factor expresivo en la moralidad, por el que llamar a algo bueno o malo es tanto como expresar los sentimientos de uno a favor, o en contra, de aquello que ha sido valorado. Y, finalmente, en la tercera etapa, siguiendo a Charles Stevenson, se añade un factor imperativo, en el que decir que aquello que fue valorado como algo bueno es tanto elogiarlo como urgir a otros a que lo hagan, o valoren de ese mismo modo (p. 43). De esta forma, una moralidad fundamentada en el «emotivismo» consistirá en que el agente se vea libre de restricciones para vivir según sus preferencias subjetivas, tratando de conseguir que otros agentes de la sociedad tengan la misma valoración subjetiva basada en sentimientos y preferencias, dependiendo del poder que pueda tener (pp. 79-88 y $117-126)$. 
Los efectos manipuladores que produce una moral fundada en el «emotivismo» se pueden observar a través de otros tres pasos, expuestos en el segundo capítulo del libro de Bello Rodríguez y Giménez Amaya. El primero es la caracterización que hace MacIntyre del «yo» de la Modernidad como un «yo» propiamente emotivista, para el que la sociedad se presenta como algo que puede oponerse a sus deseos (pp. 112-116). El segundo paso se corresponde con las relaciones que el «yo» tiene con lo que MacIntyre llama la «sociedad del individualismo burocrático», donde se revela una de las grandes paradojas de la sociedad Moderna (pp. 116-120). La estructura de tal sociedad, indica MacIntyre, se asemeja a los organismos de administración que se caracterizan por estar ocupados en la lucha competitiva por unos recursos siempre escasos que ponen al servicio de fines predeterminados. Pero en la «sociedad del individualismo burocrático» tales fines no son determinados racionalmente, sino que son establecidos por los deseos y sentimientos del sujeto. En este sentido, el «yo» emotivista no se ve limitado en aquello que desea y elige. Por el contrario, la organización burocrática le aporta los medios para alcanzar sus fines personales, y solo en la medida en que el «yo» no percibe la satisfacción eficaz de sus deseos considera su relación con la sociedad como algo problemático.

En este punto, antes de indicar el tercer paso que evidencia los efectos manipuladores del «emotivismo», se percibe ya la raíz de esta dinámica social: el «yo» emotivista puede pensar que se encuentra en su lugar natural porque las características de esta sociedad, en apariencia antagónicas, le permiten comportarse de un modo que puede satisfacer sus deseos con un mínimo de limitaciones. Sin embargo, usando la lógica del «emotivismo» antes expuesta, puede ocurrir que el individuo haya sido convencido de seguir las preferencias de alguien más, sin darse cuenta de ello. En el esquema de Spaemann introducido al principio, el sujeto se encontraría, en realidad, en una sociedad regida por una ética no-natural que no le permite escapar de sus caprichos, y lo deja a merced de los caprichos de alguien más.

Por lo indicado, el tercer paso hace referencia al poder con el que las relaciones manipuladoras se hacen evidentes a través de los perso- 
najes propios de la sociedad Moderna: el rico, el gerente y el terapeuta (pp. 120-126). En efecto, el rico es el personaje que en la sociedad Moderna se caracteriza por querer evitar cualquier tipo de aburrimiento y lo hace suscitando en los demás comportamientos que satisfagan sus propios deseos. Por otro lado, el gerente es el tipo de personaje que se encarga de gestionar medios (humanos y materiales) para alcanzar unos objetivos. Su relación con ellos es de instrumentalización y, por tanto, se encarga de borrar toda distinción entre relaciones manipuladoras y no manipuladoras. Finalmente, el terapeuta, que se encargaría de integrar a todo individuo que no se sienta parte de este proceso de manipulación. De este modo, tanto los «derechos», como la «utilidad», pasan a ser conceptos que están al servicio de los deseos manipuladores de los sujetos (pp. 126-137).

A través de lo explicado, se entiende que MacIntyre diga que las sociedades modernas están imbuidas en cultura moral emotivista (p. 43). De allí la importancia del análisis que se lleva a cabo por extenso en el segundo capítulo, en el que se muestra la visión crítica de la modernidad de MacIntyre en su libro After Virtue, donde se desarrollan las características del «emotivismo» moderno, fuertemente arraigado en el lenguaje, la cultura, y el pensamiento contemporáneo (pp. 107-142), y que hemos mostrado en los párrafos anteriores. En el resto del segundo capítulo, después de hacer una presentación de las principales características e hitos de esta obra, se ofrece una descripción sucinta de la cultura moral de la modernidad, se expone cuál fue la génesis del esquema moral moderno, y se indican cuáles fueron los intentos de la Ilustración de justificar sus planteamientos morales, tal como los entiende el profesor anglosajón (pp. 71-98).

Destaca también en el segundo capítulo del libro la idea de que uno de los grandes problemas de la Modernidad se encuentra en la falta de consenso en cuestiones éticas. Esto se debería, en gran medida, a la pérdida del contexto adecuado para la comprensión de muchos términos morales que habían tenido su referencia en una cierta «objetividad justificada», ahora inexistente (pp. 79-88). Para MacIntyre, esto ha ocurrido por un abandono del esquema teleológico que se encontraba en el pensamiento clásico. Tal esquema había funcionado por la integración de tres compo- 
nentes: [i] la concepción de una naturaleza humana ineducada; [ii] la concepción de los preceptos de una ética racional; y [iii] la concepción de una naturaleza-humana-como-podría-ser-si-se-realizara-su-telos (p. 83).

Con el abandono del tercer componente teleológico del esquema indicado, la Modernidad se habría quedado con una idea de la naturaleza humana tal como se presenta en su dimensión más naturalizada, y con unos preceptos racionales que tendrían que forjar una forma civilizada de convivencia social. Sin embargo, esto terminó por producir una visión fragmentada de los fundamentos de la vida moral a través de visiones contrapuestas de la vida práctica, que se han traducido en innumerables posturas, enfrentadas entre sí, en los intentos de determinar cuáles son, o cuáles deberían ser, los fundamentos de una Moral que busca ser imperante en la actualidad. Esta Moral intentaría, además, enfrentarse de alguna manera racional al «emotivismo» pero, al no conseguir una adecuada y unitaria fundamentación de sus propios principios, quedaría inerme frente a los ataques de los expresivistas, que la acusarían de irracional (pp. 171-177). Las características de esta Moral Moderna revelan, a juicio de MacIntyre, las dificultades provenientes de esa visión fragmentada que no da cimiento ni a la Ética en general, ni a los juicios morales en particular. Bello Rodríguez y Giménez Amaya hacen presente esto en el tercer capítulo de su libro, al analizar Ethics in the Conflicts of Modernity, y advertir que la Moral de la que venimos hablando: [1] es una moralidad secular; [2] tiene pretensiones de universalidad; [3] busca limitar los intereses particulares de los individuos y fomentar el altruismo; [4] presenta sus términos y conceptos de forma abstracta; [5] se considera superior a cualquier otro sistema moral; [6] está llena de tensiones y desacuerdos, tanto en la práctica como en la teoría (p. 153).

Por tanto, sin una concepción teleológica de la naturaleza, los intentos de justificación de las normas morales no habrían encontrado más referentes que fracciones de lo que es propiamente natural en el ser humano, ya sea el conjunto de sus pasiones, una versión reducida de su racionalidad, o una capacidad de decidir carente de otros elementos vitales (pp. 98-100). El hito intelectual que habría afianzado lo que acabamos de indicar habría sido, en el pensamiento moderno y contemporáneo, la 
proclamación de que ninguna conclusión sobre lo que debe ser hecho por el ser humano, puede ser deducida de aquello que se presenta tal como es; afirmación que lleva, además, a la idea de que los juicios morales no pueden ser considerados ni verdaderos ni falsos (pp. 101-105). La consecuencia es el dominio del «emotivismo», que promueve un individualismo radical que proclama que nada puede limitar al sujeto incluida, por supuesto, la misma sociedad cuya fundamentación se ha vuelto incomprensible en el mundo moderno (pp. 112-116).

Las críticas a la Modernidad por parte de Alasdair MacIntyre evidencian su perspectiva aristotélica. Sin embargo, nuestro pensador moral no tuvo una predilección por la filosofía clásica en el inicio de su vida académica. Éste pasó por la indecisión, intelectual y vital, de inclinarse o hacia el pensamiento aristotélico, o hacia el expresivismo emotivista. Toda esta cuestión tomó nuevos matices cuando se aproximó al marxismo. En efecto, durante sus años universitarios en el Queen Mary College, y a través de algunos compañeros de la universidad, MacIntyre se incorporó al Partido Comunista británico, que abandonó después, sobre todo, por su ineficacia como organización, y la poca coherencia que mostraban sus partidarios frente a las atrocidades del marxismo soviético (p. 32). Muestra clara del rechazo de la filosofía marxista hacia determinados bienes espirituales propios del ser humano, y sin los cuales no es posible comprenderlo en su integridad.

Pero su paso por el estudio de esta filosofía le ayudó a resolver algunos conflictos en los que se encontraba atrapado. Desde el pensamiento de Marx, MacIntyre llega al convencimiento de la raíz ideológica del liberalismo, entendido como máscara mistificadora de ciertos intereses sociales, y que obra un dominio silencioso sobre la sociedad en nombre de la misma libertad que dice defender. Entiende, además, con acierto -tal como se indica también en el tercer capítulo del libro- que la sociedad Moderna es capitalista y tecnológica, y que ésta busca que los seres humanos configuren sus deseos a partir de las exigencias del mercado y del flujo de dinero. Esto no lleva al individuo a elegir lo bueno, sino que sirve a los intereses de ciertas élites económicas. La gente no advierte esto porque piensa que el mercado le ofrece a la sociedad una actividad individual 
y libre (pp. 141-159). Pero esa visión es errónea, ya que tal dominio se da por medio de la disolución de lazos humanos tradicionales que son empobrecidos por la falta de comprensión de la propia vida como búsqueda y logro del bien humano (p. 33).

Pero la enseñanza más significativa que Alasdair MacIntyre obtiene de su etapa marxista, para su itinerario intelectual, fue entender que toda forma de moralidad, incluida la del liberalismo moderno, por más universal que pueda declararse, pertenece a un grupo social específico, y es el producto de la vida y de la historia de aquel grupo. El filósofo escocés llega a entender que la moralidad no existe sino en formaciones sociales reales o posibles, y eso que expresa, o puede expresar, coincide con sus formas realizadas socialmente (p. 34 ).

De acuerdo con «Valoración ética de la Modernidad según Alasdair MacIntyre», parece que es posible afirmar que la filosofía de Marx le mostró al filósofo británico las bases para comprender la Moral Moderna. Como se ha advertido, el hecho de que MacIntyre lo haya aprendido de este modo es una cuestión de su itinerario vital e intelectual. Sin embargo, parece consistente afirmar que esto se pudo dar, también, por la manera como el marxismo lleva a cabo su aproximación racional a la vida práctica, y que marca un contraste con los debates académicos de la actualidad. De una forma cercana a como lo que hace Aristóteles en sus obras filosóficas sobre la moralidad y la vida política, Marx busca reflexionar sobre la vida humana teniendo como punto de partida los bienes de quienes viven en una sociedad regida por el intercambio económico, y el modo como ésta está organizada. Esto, como se ve en los apartados finales del tercer capítulo del libro, tiene una fuerte influencia en la manera en que MacIntyre habla de la «ética-del-mercado» y la «ética-del-Estado» y cómo describe que los individuos puedan ceder en sus deseos frente a las exigencias de ambos tipos de derivaciones de la Moral Moderna (pp. 165-170). La conclusión de MacIntyre es que cualquier otra manera de abordar las discusiones éticas o morales es susceptible de estancarse en posiciones abstractas e irreconciliables, como ocurre con frecuencia en los debates contemporáneos que implican principios antagónicos, tal como se da en 
el enfrentamiento entre utilitaristas y kantianos, y sus formas contractualistas (pp. 36-38, 59-62 y 171-177).

Lo indicado más arriba, lleva a MacIntyre, posteriormente, a afirmar el papel secundario de la filosofía moral académica como teoría, con respecto a la praxis moral, ya que solo en la actividad humana es posible distinguir entre los deseos personales subjetivos, y lo que es bueno desear por sí mismo (pp. 51-52). Por tanto, para entender el enfrentamiento entre la Moral y el «emotivismo», es importante no desarraigar esta discusión de su contexto histórico, y de su actual realización en la vida práctica tanto individual como social.

En efecto, para MacIntyre, siguiendo al Estagirita, la reflexión sobre los bienes humanos es la que conduce consecuentemente a la indagación sobre las mejores actividades para alcanzarlos, y a la determinación del modo para que tales actividades pueden ser preservadas en la vida social. Por tanto, el sentido de lo que es bueno hacer, o de lo que es bueno llevar a cabo, teniendo como fin el bien, se da propiamente en el contexto social de una ética aristotélica de las virtudes, en la que éstas representan los modos más excelentes de alcanzar los bienes morales, que son propios de los seres racionales (pp. 42-61). La aspiración moral del sujeto no se daría ya en la simple expresión de sus propias valoraciones, o de sus preferencias subjetivas, sino en alcanzar aquello que es propiamente humano.

Por tanto, en su trayectoria vital, Alasdair MacIntyre termina por alejarse de las posturas teóricas de la Modernidad después de una búsqueda denodada por alcanzar una cierta coherencia en sus investigaciones filosóficas (pp. 41-42). Esto le lleva a duras críticas al sistema de la filosofía moral académica contemporánea, a la que tilda de estar divorciada del estudio de la praxis moral, de estar centrada en los aspectos teóricos de lo estrictamente curricular, y de haber decaído al convertirse en una disciplina que promueve un status quo en el que las normas de los trabajos académicos se rigen por la ley de publicar a toda costa una serie de escritos que no serán leídos. La consecuencia final es el refuerzo de un conformismo poco crítico con la misma Modernidad que ha impuesto esta forma de trabajar en la filosofía (pp. 69-72). 
Para terminar, la trayectoria intelectual de MacIntyre no puede estar completa sin la consideración de su aceptación del pensamiento de Tomás de Aquino como una forma de adoptar un aristotelismo que va más allá de Aristóteles, y de su conversión al catolicismo (pp. 62-68). Estos detalles son fundamentales para entender por qué para nuestro autor la filosofía aristotélico-tomista presenta los elementos adecuados para alcanzar una comprensión de la narrativa que los agentes necesitan para superar los distintos conflictos morales que la Modernidad ha dejado sin resolver (pp. 184-191). Ésta, con algunos aportes de Marx, concluye el libro, representa para él la mejor postura teórica en los debates contemporáneos de la filosofía moral académica, ya que atiende a los elementos esenciales de la vida práctica.

\section{Referencias}

Bello Rodríguez, H. J. \& Giménez Amaya, J. M. 2018. Valoración ética de la modernidad según Alasdair MacIntyre. Pamplona: EUNSA.

MacIntyre, A. 1987. Tras la virtud. Barcelona: Crítica.

Spaemann, R. 2010. Ética. Cuestiones fundamentales. Pamplona: EUNSA. 
